\title{
1 Rgs12 enhances osteoclastogenesis by suppressing Nrf2 activity and 2 promoting the formation of reactive oxygen species
}

3 Andrew $\mathrm{YH} \mathrm{Ng}^{1,2,4, a, b}$, Ziqing $\mathrm{Li}^{1}$, Megan M Jones, $\mathrm{PhD}^{2}$, Chengjian Tu, $\mathrm{PhD}^{3,4}$, Merry J Oursler,

$4 \quad \mathrm{PhD}^{5}$; Jun Qu, Ph.D. ${ }^{3,4}$, Shuying Yang, MD, $\mathrm{PhD}^{1, a, b, *}$

6 'Department of Anatomy and Cell Biology, School of Dental Medicine, University of

7 Pennsylvania, Philadelphia, PA

8 'Department of Oral Biology, School of Dental Medicine, University at Buffalo, Buffalo, NY

$9{ }^{3}$ Department of Pharmaceutical Science, School of Pharmacy and Pharmaceutical Sciences,

10 University at Buffalo, NY

$11{ }^{4}$ New York State Center of Excellence in Bioinformatics and Life Sciences, Buffalo, NY

$12{ }^{5}$ Division of Endocrinology, Metabolism, Nutrition, and Diabetes, Mayo Clinic, Rochester, MN,

13 USA

15 a National Institute of Arthritis and Musculoskeletal and Skin Diseases (NIAMS)

$16{ }^{\mathrm{b}}$ National Institute of Aging (NIA)

18 *Corresponding author:

19 Shuying Yang, M.D., Ph.D.

20 Associate Professor

21 University of Pennsylvania

22 Department of Anatomy and Cell Biology

23 Levy 443,240 S. $40^{\text {th }}$ St

24 Philadelphia, PA 19104

25 Phone: (215) 898-2685

26 Email: shuyingy@upenn.edu 
$1 \quad$ Rgs12 enhances osteoclastogenesis by suppressing Nrf2 activity and promoting the

2 formation of reactive oxygen species

3 Andrew $\mathrm{YH} \mathrm{Ng}^{1,2,4}$, Ziqing $\mathrm{Li}^{1}$, Megan $\mathrm{M} \mathrm{Jones}^{2}$, Chengjian $\mathrm{Tu}^{3,4}$, Jun $\mathrm{Qu}^{3,4}$, Merry J Oursler ${ }^{5}$

4 Shuying Yang ${ }^{1 *}$

$6{ }^{1}$ Department of Anatomy and Cell Biology, School of Dental Medicine, University of

7 Pennsylvania, Philadelphia, PA

$8{ }^{2}$ Department of Oral Biology, School of Dental Medicine, University at Buffalo, Buffalo, NY

$9{ }^{3}$ Department of Pharmaceutical Science, School of Pharmacy and Pharmaceutical Sciences,

10 University at Buffalo, NY

$11{ }^{4}$ New York State Center of Excellence in Bioinformatics and Life Sciences, Buffalo, NY

13 *Corresponding author: Dr. Shuying Yang, shuyingy@upenn.edu, Department of Anatomy and

14 Cell Biology, School of Dental Medicine, University of Pennsylvania, Philadelphia, PA.

\section{ABSTRACT}

17 The Regulator of G-protein Signaling 12 (Rgs12) is important for osteoclast (OC) differentiation, 18 and its deletion in vivo protected mice against pathological bone loss. To characterize its 19 mechanism in osteoclastogenesis, we selectively deleted Rgs12 in OC precursors using the

20 LysM-Cre transgenic line or overexpressed the gene in RAW264.7 cells. Rgs12 deletion led to

21 increased bone mass with decreased OC numbers, whereas its overexpression increased OC

22 number and size. Proteomics analysis of Rgs12-deficient OCs identified an upregulation of

23 antioxidant enzymes under the transcriptional regulation of $\mathrm{Nrf2}$, the master regulator of

24 oxidative stress. We confirmed an increase of Nrf2 activity and impaired production in Rgs12-

25 deficient cells. Conversely, Rgs12 overexpression suppressed Nrf2 through a mechanism

26 dependent on the $26 \mathrm{~S}$ proteasome, and promoted RANKL-induced phosphorylation of ERK1/2

27 and NFKB, which was abrogated by antioxidant treatment. We therefore identified a novel role

28 of Rgs12 in regulating Nrf2, thereby controlling cellular redox state and OC differentiation.

\section{INTRODUCTION}


1 Osteoporosis is a pervasive disorder characterized by skeletal fragility and microarchitectural

2 deterioration that predisposes individuals to bone fractures. The disease has a significant global

3 impact, affecting an estimated 200 million people worldwide and exerts a heavy economic

4 burden - the affected are projected to rise by approximately $50 \%$ within the next 10 years (1).

5 Therefore, understanding the pathogenesis of osteoporosis is an urgent matter to develop better

6 treatments for this debilitating disease.

8 Bone remodeling is carried out by the coordinated actions of the bone-forming osteoblasts

9 (OBs) and the bone-resorbing osteoclasts (OCs). Disorders of skeletal deficiency, such as

10 osteoporosis, are typically characterized by enhanced osteoclastic bone resorption relative to

11 bone formation, thereby resulting in net bone loss. Although significant progress has been made

12 in OC behavior, it remains largely unknown about the regulatory mechanism that drives OC

13 differentiation, which restricts the effectiveness of current treatments.

15 Regulators of G-protein Signaling (RGS) are a family of proteins comprised of more than 30

16 proteins that share a conserved RGS domain and play a classical role in attenuating G protein-

17 coupled receptor (GPCR) signaling through its GTPase-accelerating protein (GAP) activity to

18 inactivate the $\mathrm{Ga}$ subunit $(2,3)$. The RGS proteins are multifunctional proteins which hold vital

19 cellular processes, including cell differentiation. Rgs12 is unique in that it is the largest protein in

20 its family. In addition to the RGS domain, contains a PSD-95/Dlg/ZO1 (PDZ) domain, a

21 phosphotyrosine-binding (PTB) domain, a tandem ras-binding domain (RBD1/2), and a GoLoco

22 interaction motif. The multi-domain architecture of Rgs12 suggests a role as a scaffolding

23 protein in complexes where multiple signaling pathways might converge (4-8).

25 Reactive oxygen species (ROS) are produced as a normal byproduct of cellular metabolism (9).

26 Recent studies clearly show that RANKL-induced reactive oxygen species (ROS) are 
1 indispensable for OC differentiation (9-12). ROS at high levels induce oxidative stress, which if

2 left unchecked becomes deleterious to cell. At low concentrations, however, ROS have been

3 shown to participate in signaling events in OCs, including the RANKL-dependent activation of

4 mitogen-activated protein kinases (MAPKs), phospholipase C gamma (PLCY), nuclear factor

5 kappa B (NFKB), and $\left[\mathrm{Ca}^{2+}\right]$ oscillations; all of which contribute to the activation of nuclear factor

6 of T-cells (NFAT), the master regulator of OC differentiation. Furthermore, multiple lines of

7 evidence have consistently shown that suppression of ROS by various means inhibits OC

8 differentiation (10-12). In particular, RANKL-dependent activation of PLCY, [Ca ${ }^{2+}$ oscillations,

9 and NFAT were abrogated when the OC precursors were treated with the antioxidant N-

10 acetylcysteine (NAC) (11). Furthermore, our previous studies have that Rgs12 silencing could

11 inhibit PLCY activation, $\left[\mathrm{Ca}^{2+}\right]$ oscillations, and the expression of NFATc1 and its downstream

12 factors (13). Hence, these findings led us to hypothesize that Rgs12 may play a role in

13 regulating the cellular redox state, thereby controlling OC differentiation.

15 Using an in vivo Rgs12 conditional knockout mouse model, we found that RANKL-dependent

16 ROS was suppressed in Rgs12-deficient bone marrow macrophages (BMMs). Additionally,

17 Rgs12-deficient cells have elevated levels of phase II detoxification and antioxidant enzymes.

18 We further identified that Rgs12 deficiency increased the activation of Nrf2, the master

19 transcription factor responsible for the expression of antioxidant proteins. Conversely, Rgs12

20 overexpression could suppress Nrf2 and promote osteoclastogenesis. Our results therefore

21 demonstrate a novel function of Rgs12 in regulating ROS during OC differentiation, likely by

22 suppressing Nrf2 to inhibit the expression of antioxidant proteins. These findings contribute to a

23 better understanding of ROS regulation in OCs and future treatment strategies for diseases of

24 excessive bone loss such as osteoporosis.

\section{RESULTS}


Targeted deletion of Rgs12 in myeloid cells increased trabecular bone mass.

2 To assess the role of Rgs12 on OC differentiation and bone remodeling in vivo, we generated a

3 conditional gene knockout mouse model by crossing Rgs $12^{\text {flox/flox }}$ mice with LysM-Cre transgenic

4 mice (LysM;Rgs12 $\left.2^{f / f l}\right)$. The LysM promoter-driven Cre expression targets the gene deletion to

5 cells of the myeloid lineage, including monocytes/macrophages $(14,15)$. The Cre-lox-mediated

6 deletion of the Rgs12 gene was confirmed by PCR amplification of spleen genomic DNA (Fig.

7 1A), and qPCR to measure Rgs 12 transcripts in isolated BMMs (Fig. 1B) thereby confirming our

8 mouse $R g s 12$ conditional gene knockout model.

10 LysM;Rgs12 $2^{\mathrm{fl} / f \mathrm{l}}$ mice exhibited an increase in trabecular bone mass, evident in the H\&E-stained

11 bone sections of the proximal tibia (Fig. 1C) and micro-computed tomography (micro-CT)

12 analysis of the femoral trabecular bone morphology and microarchitecture (Fig. 1D and E).

13 Quantitative micro-CT measurements further revealed significant increases in both trabecular

14 number (Tb.N) and thickness (Tb.Th), and reduced trabecular separation (Tb.Sp) (Fig. 1G-I).

15 Our results therefore demonstrate the importance of Rgs12 in bone remodeling.

17 Rgs12 promotes osteoclast formation.

18 To assess the role of Rgs12 in osteoclastogenesis, OC precursors isolated from LysM;Rgs $12^{\mathrm{fl} / \mathrm{fl}}$

19 mice were differentiated using M-CSF and RANKL. While control BMMs differentiated into large,

$20 \mathrm{TRAP}^{+}$multinucleated OCs, Rgs12-deficient precursor cells showed a reduction in the number

21 of OCs containing 6-9 nuclei and 10+ nuclei, which were also visibly smaller (Fig. $2 \mathrm{~A}$ and B).

22 Complementing our Rgs12 knockout model, we generated an Rgs12 overexpression OC model

23 in which RAW264.7 cells were stably-transfected with a vector carrying a recombinant N-

24 terminus FLAG-tagged Rgs12 gene (Flag-Rgs12). Rgs12 overexpression in RAW264.7 cells

25 was confirmed by western blotting (Fig. 2C). Using this cell model, we next determined whether

26 Rgs12 overexpression could promote osteoclastogenesis (Fig 3D). Converse to our findings in 
1 Rgs12 knockout cells, we found that overexpression of Rgs12 in RAW264.7 cells led to an

2 increased number of OCs with 10+ nuclei (Fig. 3E). We also observed significantly decreased

3 numbers of smaller OCs containing 3-5 and 6-9 nuclei in Rgs12 overexpressing cells (Fig. 2E),

4 presumably because most of the smaller OCs have fused to form large OCs containing $10+$

5 nuclei. In a study investigating the relationship between osteoclast size and state of resorptive

6 activity, a greater proportion of large osteoclasts were active whereas non-resorbing osteoclasts

7 were on average smaller (16). Additionally, quantification of the mean areas of OCs with $10_{+}$

8 nuclei revealed that Rgs12-overexpressing OCs were larger as compared to empty vector-

9 transfected controls (Fig. 2F). Our findings consequently demonstrate the importance of Rgs12

10 in promoting $\mathrm{OC}$ formation, which is consistent with the osteopetrotic phenotype observed in

11 Rgs12-deficient mouse model.

\section{Rgs12-defcient osteoclast precursors show an increased expression of Nrf2-dependent}

\section{4 antioxidant proteins.}

15 To uncover the role of Rgs12 in OC differentiation, we employed the IonStar liquid

16 chromatography tandem mass spectrometry (LC-MS/MS)-based quantitative proteomics

17 strategy (17) to profile the temporal dynamics in the global protein levels in $\mathrm{Rgs} 12^{\mathrm{fl} / \mathrm{fl}}$ and

18 LysM;Rgs12 $2^{f / / 1 / 1}$ BMMs at 0, 1, 3, and 5 days of OC differentiation (Fig. 4). Proteomics analysis

19 identified 3,714 quantifiable proteins that are present in all samples (no missing data), using a

20 highly stringent identification criteria of $\geq 2$ peptides per protein and $1 \%$ false discovery rate (Fig.

$213 \mathrm{~A})$. Within this dataset, we identified 83 and 61 unique proteins that were significantly up- and

22 downregulated, respectively, in LysM;Rgs12 $12^{\mathrm{fl/fl}} \mathrm{BMMs}$ relative to $\mathrm{Rgs} 12^{\mathrm{fl} / \mathrm{fl}} \mathrm{BMMs}$. Proteins were

23 considered significantly altered if they exceeded the thresholds set at $p<0.05$ and $>0.3 \log _{2^{-}}$

24 transformed ratio (Fig. 3B). Most of the protein expression changes in Rgs12-deficient cells

25 were captured at 3 and 5 days of OC differentiation (Fig. 3A). To determine the biological

26 significance of these altered proteins, we performed gene ontology analysis to identify the 
1 canonical pathways involved (Fig. 3C). Processes related to OC differentiation (e.g. "NFAT

2 Signaling", "RANK Signaling in OCs", and "Role of OCs in Rheumatoid Arthritis") were enriched

3 at 3 and 5 days of $O C$ differentiation. Closer inspection showed that OC marker proteins

4 including metalloproteinase-9 (Mmp9), tartrate-resistant acid phosphatase (Trap), ATPase $\mathrm{H}^{+}$

5 transporting V0 subunit D2 (Atp6v0d2), and integrin $\beta 3$ (Itgb3) were significantly downregulated

6 in LysM;Rgs12 $2^{f / f \mid l}$ OCs (Fig. 3D). Additionally, the analysis revealed several biological functions

7 related to ROS homeostasis that were impacted by Rgs12 deletion (e.g. "Production of ROS",

8 "Superoxide Radical Degradation", and NRF2-mediated Stress Response") (Fig. 3C). Inspection

9 of the proteins involved in these pathways showed a significant upregulation of numerous Nrf2-

10 dependent antioxidant enzymes responsible attenuating oxidative stress, including:

11 peroxiredoxin 1/4 (Prdx1/4), thioredoxin 1/2 (Trxr1), glutathione reductase (Gshr), and NAD(P)H

12 dehydrogenase quinone 1 (Nqo1) (Fig. 3E). Upstream regulator (transcription factor) analysis

13 identified that the antioxidant enzymes upregulated in LysM;Rgs12 $2^{\mathrm{f} / \mathrm{fl}}$ OCs share the common

14 upstream regulator Nrf2, a key transcription factor that regulates cellular redox balance through

15 the expression of protective antioxidant and phase II detoxification proteins. $(18,19)$ (Fig S4A).

16 Although the upstream regulator analysis predicted an upregulation of Nrf2 activity, the

17 transcription factor itself was not detected by our proteomics analysis. Proteins of typically low

18 abundance such as cytokines, signal regulatory molecules, and transcription factors tend to be

19 "crowded out" during MS analysis by more highly abundant proteins such those proteins

20 involved in glycolysis and purine metabolism, protein translation, and cytoskeletal components

21 (20). Nonetheless, our proteomics-based discovery tool allowed us to generate the hypothesis

22 that Nrf2 is aberrantly activated by Rgs12 deletion, causing excessive clearance of ROS by

23 antioxidant enzymes and in turn disrupting OC differentiation.

25 Deletion of Rgs12 elevated Nrf2/Keap1 expression and Nrf2 activity 
1 Based on our proteomics analysis, we hypothesized that Rgs12 is needed to suppress Nrf2

2 activity and facilitate the formation of ROS, which has been previously shown to play a critical

3 role in OC differentiation $(10,21)$. To test this hypothesis, we assessed Nrf2 activity and the

4 expression of Nrf2 and Keap1 in Rgs12 f//fl and LysM;Rgs12f//fl BMMs (Fig. 4). Western blotting of

5 Nrf2 in day 3 OCs also showed increased levels of Nrf2 in LysM;Rgs12 $2^{\mathrm{fl} / \mathrm{fl}}$ cells (Fig. 4A and B).

6 Keap1, however, which is known to suppresses Nrf2 activity by facilitating its degradation via

7 the proteasome pathway, was unexpectedly elevated in Rgs12-deficient cells. Furthermore,

8 immunofluorescence staining of Nrf2 demonstrated increased nuclear translocation of the

9 transcription factor in LysM;Rgs12 $2^{\text {fl/fl }}$ cells (Fig. 4C, upper panel). The elimination of ROS with N-

10 acetylcysteine (NAC), a precursor to the antioxidant glutathione, was able to completely

11 suppress Nrf2 nuclear translocation in both Rgs12f//fl and LysM;Rgs12 $12^{\mathrm{fl} / \mathrm{fl}}$ BMMs (Fig. 4C, middle

12 panel). Conversely, induction of oxidative stress using the peroxide tert-buthylhydroxyperoxide

13 (tBHP) potently induced Nrf2 nuclear translocation (Fig. 4C, bottom panel). To further test

14 whether elevated Nrf2 activity in Rgs12-deficient OCs could result in reduced intracellular ROS

15 levels, we detected intracellular ROS levels. As expected, the RANKL-dependent ROS

16 induction observed in control cells was suppressed in LysM;Rgs12 $2^{\mathrm{fl} / \mathrm{fl}}$ OCs (Fig. 4D). These

17 findings demonstrate an abnormal upregulation of Nrf2 activity and expression in Rgs12-

18 deficient cells, indicating that Rgs12 may be required to suppress Nrf2 to facilitate

19 osteoclastogenesis.

21 Rgs12-mediated suppression of Nrf2 activity is dependent on the proteasome

22 degradation pathway.

23 Under basal conditions (i.e. absence of cellular stress), Nrf2 remains inactive through its

24 interaction with Keap1, which causes its continual ubiquitination and degradation via the

25 proteasome pathway $(22,23)$. A variety of stress conditions can induce conformational changes

26 in Keap1, thereby releasing Nrf2 from the ubiquitin-proteasome pathway, allowing it to 
1 accumulate and translocate into the nucleus $(24,25)$. To better understand the mechanism by

2 which Rgs12 suppresses Nrf2 activity, we therefore first determined whether the ability of Rgs12

3 to suppress Nrf2 activity relies on this canonical mechanism (Fig. 5A). Given that Rgs12

4 deletion resulted in elevated Nrf2 expression and nuclear translocation, we first determined

5 whether Rgs12 overexpression could exert the opposite effect (Fig. 5A and B). We measured

$6 \quad$ Nrf2 protein levels in RAW264.7 cells stably transfected with the Rgs12-His or empty vector and

7 found no difference when cells are at their basal state (i.e. uninduced). Stimulation of

8 RAW264.7 cells with tert-buthylhydroquinone (tBHQ), which is known to directly bind Keap1 and

9 attenuate its inhibitory effect on Nrf2 (26), caused a robust induction of Nrf2 protein levels in a

10 dose-dependent manner (Fig. 5A and B). More importantly, RAW264.7 cells overexpressing

11 Rgs12 showed a significant reduction of Nrf2 protein levels that resulted from Keap1 inhibition

12 compared to those in the control cells. Moreover, the ability of Rgs12 to facilitate Nrf2

13 degradation despite the inhibition of Keap1 suggests that Rgs12 functions downstream of

14 Keap1, either by controlling the ubiquitination or proteasomal degradation of Nrf2.

16 Given the possibility that the reduction of Nrf2 levels in Rgs12 overexpression cells may be a

17 result of increased Nrf2 degradation, we further tested whether inhibiting the proteasome, a step

18 downstream of Keap1, could attenuate the ability of Rgs12 to facilitate Nrf2 degradation (Fig. 5D

19 and $\mathrm{E})$. Similar to $\mathrm{tBHQ}$, preventing Nrf2 degradation using the proteasome inhibitor MG-132

20 caused Nrf2 protein to substantially accumulate (Fig. 5D, left panel). Interestingly, when Nrf2

21 protein levels were artificially induced, we observed the presence of a lower molecular weight

22 band, which could correspond to a different post-translational modification state (e.g.

23 unphosphorylated or non-ubiquitinated). Furthermore, we did not observe any changes in

24 Keap1 protein levels. In the previous scenario wherein Rgs12 overexpression could still

25 promote Nrf2 degradation in spite of $t B H Q$ treatment, this was not the case when using MG-

26 132. In fact, inhibiting the proteasome was able to reverse the ability of Rgs12 to promote Nrf2 
1 degradation, indicating its requirement for the proteasome's function. Repeating this experiment

2 in RAW264.7 cells differentiated for 3 days with RANKL showed that Nrf2 levels were

3 suppressed in OCs (Fig. 5D, right panel); likely due to reduced transcriptional activity, which

4 corroborates with findings from previous studies (Fig. 5F) (21, 27). More importantly, Rgs12

5 overexpression could suppress Nrf2 protein levels, but inhibition of the proteasome using MG-

6132 reversed this effect (Fig. 5D, right panel). To confirm that Rgs12 inhibits Nrf2 through a

7 post-translational mechanism, we measured Nrf2 transcript levels by qPCR and found no

8 difference between wild-type or Rgs12-overexpressing cells (Fig. 5F). Overall, our data

9 collectively indicate that Rgs12 suppresses Nrf2 activity by facilitating its degradation through

10 the proteasome-dependent pathway.

\section{Rgs12-mediated activation of osteoclast MAPK and NFKB signaling is dependent on}

\section{3 intracellular ROS.}

14 It was previously demonstrated that ROS could act as an intracellular signal mediator OC

15 differentiation, and is required for the RANKL-dependent activation of p38 mitogen-activated

16 protein kinase (MAPK), extracellular signal-regulated kinase (ERK), and NFKB (10, 28). Given

17 our findings that Rgs12 could suppress the activity of Nrf2 and thereby promoting intracellular

18 ROS, we hypothesized that Rgs12 could promote RANKL-dependent signaling, and that this

19 effect would be abrogated by the addition of an antioxidant (Fig. 6A and B). As expected,

20 RAW264.7 cells overexpressing Rgs12 demonstrated a more robust activation of ERK1/2 and

21 NFKB phosphorylation but not p38 MAPK. Pretreating Rgs12 overexpressing cells with the

22 antioxidant NAC diminished ERK1/2 activation, and almost completely abrogated NFKB

23 activation. These results support the role of Rgs12 in promoting ROS that is important OC

24 signaling, likely through the suppression of Nrf2 activity.

\section{DISCUSSION}


1 The importance of ROS in osteoclasts has been underlined by the growing body of evidence

2 that ROS increased with aging or during inflammation can stimulate bone resorption and

3 exacerbate bone loss (9). Targeting ROS in diseases of excess bone resorption such as

4 osteoporosis could therefore be an important therapeutic strategy. Additionally, an important

5 mechanism of cellular ROS clearance relies on the Keap1-Nrf2 pathway which is very well

6 characterized, especially in the context of cancer biology (29). However, the upstream signaling

7 molecules that could regulate the Keap1-Nrf2 axis in OCs remains unknown. Targeting this gap

8 in knowledge, our study uncovered a novel role of the signaling protein Rgs 12 in regulating

9 Nrf2, thereby controlling cellular redox state and OC differentiation (Fig. 6C).

11 In this study, we demonstrated the essential role of Rgs12 in OC differentiation such that Rgs12

12 knockout mice exhibited increased bone mass (Fig. 1) and OC precursors isolated from these

13 mice showed reduced OC differentiation (Fig. 2). On the contrary, overexpressing Rgs12 in

14 RAW264.7 cells significantly promoted OC formation and increased the size of resultant OCs.

15 However, the mechanism by which Rgs12 regulates OC differentiation remains unclear.

17 Proteomics is a powerful tool that has led to numerous discoveries of proteins and biological

18 processes that drive OC differentiation (30). Notably, this technique was recently used to map

19 the podosome proteome which helped to advance our understanding of determinants in the

20 macrophage multinucleation process $(31,32)$, and how metabolism and energy is redirected

21 towards bone resorption in OCs (33). Proteomics can therefore provide a broad yet informative

22 overview of the systemic changes in the differentiating OC. To discover the cellular function of

23 Rgs12 in OCs, we employed a robust and high-throughput quantitative proteomics approach to

24 characterize the global protein changes of OCs derived from Rgs $12^{f / / f l}$ and LysM;Rgs $12^{f / / f l}$ BMMs

25 (Fig. 3). Interestingly, the analysis identified the upregulation of a collection of antioxidant

26 enzymes that are transcriptionally regulated by the antioxidant response element (ARE) in the 
1 promoter region, which is activated by the transcription factor Nrf2 (34). Based on this evidence,

2 we further investigated the role of Rgs12 in Nrf2 signaling. We found that Nrf2 protein levels and

3 nuclear translocation were increased in OC precursors in which Rgs12 was deleted (Fig. 4).

4 Because our data showed that Rgs12 deficiency upregulated Nrf2, we expected that Keap1

5 levels should be reduced in order to facilitate the increased Nrf2 activity. On the contrary, Keap1

6 levels were upregulated in Rgs12-deficient cells, suggesting that the Nrf2 upregulation may be

7 independent of the Keap1. We speculate that Rgs12-deficient cells may be overcompensating

8 Keap1 expression in order to rein back the increased Nrf2 activity. Nevertheless, consistent with

9 the upregulation of Nrf2 and its corresponding antioxidant enzymes, the RANKL-dependent

10 induction of ROS was attenuated in Rgs12-deficient cells. Taking an opposite approach, we

11 demonstrated that Rgs12 overexpression in OC precursors could also enhance RANKL-

12 mediated activation of ERK and NFKB, which is known to be dependent on ROS (Fig. 7).

13 Inhibition of intracellular ROS blocked the effect of Rgs12 overexpression, indicating that Rgs12

14 promotes RANKL-dependent signaling by facilitating ROS production. Overall, the data

15 collectively demonstrate that Rgs 12 promotes osteoclastogenesis by facilitating ROS generation

16 through the suppression of Nrf2 and its target antioxidant genes.

18 Nrf2 is constitutively expressed but its activity is inhibited through its interaction with Keap1.

19 Under basal conditions, Nrf2 is restricted to the cytoplasm where it is continually depleted

20 through the proteosomal degradation pathway. When bound to Nrf2, Keap1 recruits the Cul3-

21 dependent E3 ubiquitin ligase complex, which ubiquitinates and targets Nrf2 for degradation by

22 the $26 \mathrm{~S}$ proteasome $(23,24,35,36)$. The Keap1 protein containing multiple reactive cysteine

23 residues that serve as redox sensors (26) Stressor conditions including oxidative stress causes

24 the electrophilic modification of Keap1, inducing conformational changes which cause the

25 protein to dissociate from Nrf2 and allow the transcription factor to translocate into the nucleus

26 (22). We therefore determined how Rgs12 regulates this well-defined mechanism (Fig. 5). tBHQ 
1 is a selective inhibitor of Keap1 activity by covalently binding the protein's reactive thiols and

2 could activate Nrf2 and its downstream proteins in RAW264.7 cells (26). Furthermore, tBHQ

3 inhibited OC differentiation via the upregulation of heme oxygenase-1, a Nrf2-dependent

4 antioxidant enzyme (Yamaguchi et al., 2014). In our study, we determined whether Rgs12 could

5 suppress the tBHQ-dependent upregulation of Nrf2 (Fig. 5B). We reasoned that if Rgs12 relies

6 on a Keap1-dependent mechanism, then the inhibition of Keap1 by tBHQ should prevent the

7 ability of Rgs 12 to suppress Nrf2. However, we observed that Rgs 12 was still able to suppress

8 Nrf2 despite blocking Keap1 activity, indicating that Rgs12 functions downstream of Keap1.

9 Following Keap1-mediated ubiquitination of Nrf2, the targeted protein is degraded by the

10 proteasome. Again, we reasoned that if Rgs12 is dependent on the proteasomal degradation

11 pathway, then inhibition of this pathway using MG-132 should prevent Rgs12-mediated

12 suppression of Nrf2. Indeed, we found that Inhibiting the proteasome was able to reverse the

13 Rgs12-mediated degradation of Nrf2, which places Rgs12 in between Keap1 and the

14 proteasome in the Nrf2 degradation pathway (Fig. 5D and E). Thus, Rgs12 could either regulate

15 the Cul3-dependent E3 ubiquitin ligase complex to facilitate the ubiquitination of Nrf2, or directly

16 control proteasome activity. It is interesting to note that NFKB activation is also dependent on

17 the proteasomal degradation of inhibitor of $K B(I K B)$, which otherwise sequesters NFKB to the

18 cytoplasm (37). If Rgs12 could modulate proteasome activity, it is possible that Rgs12 could

19 directly promote NFKB by facilitating the degradation of IKB. However, the fact that antioxidant

20 treatment to suppress ROS could almost completely block the phosphorylation of NFKB points

21 to an important role of ROS, and not just the proteasomal degradation of IKB in NFKB activation

22 (Fig. 6). This potential crosstalk between the NFKB and Nrf2 pathways will need to be evaluated

23 in future studies.

25 In conclusion, our study points to a novel role of Rgs12 in OC redox biology, thus forming the 26 molecular basis for developing therapies for osteoporosis and other diseases of bone loss. 
1 Additionally, we found a new factor that could modulate the Nrf2-Keap1 pathway, which is

2 important within the context of ROS biology.

\section{METHODS}

5 Generation of Rgs12 Conditional Knockout Mice. Rgs12 $12^{f / f l}$ mice were crossed with LysM-Cre

6 transgenic mice to generate Rgs12 conditional knockout mice specific to the myeloid lineage

7 (LysM;Rgs12 $\left.2^{\mathrm{fl} / \mathrm{fl}}\right)$. The methodology for generating Rgs $12^{\mathrm{fl} / \mathrm{fl}}$ and LysM-Cre mice and genotyping

8 are previously described $(15,38,39)$. Mice used for experiments were 6-8-weeks-old. All animal

9 studies were approved by the University at Buffalo Institutional Animal Care and Use Committee

10 (IACUC).

12 Histology and quantitative micro-CT measurements. Mouse femurs were excised, fixed for

$1324 \mathrm{~h}$ in $10 \%$ natural buffered formalin, and decalcified in $10 \%$ EDTA for $1-2$ weeks at $4{ }^{\circ} \mathrm{C}$. The

14 samples were embedded in paraffin and sectioned at $5 \mu \mathrm{m}$ and stained with $\mathrm{H} \& \mathrm{E}$. A quantitative

15 analysis of the gross bone morphology and microarchitecture was performed using a micro-CT

16 system (USDA Grand Forks Human Nutrition Research Center, Grand Forks, ND, USA). Fixed

17 femur from Rgs12 control and mutant mice were analyzed and 3D reconstruction was used to

18 determine bone volume to tissue volume (BV/TV), structure model index (SMI), trabecular

19 thickness (Tb.Th, $\mu \mathrm{m})$, trabecular number (Tb.N, /mm), and trabecular separation (Tb.Sp, $\mu \mathrm{m})$.

21 Generation of Rgs12 expression vectors. Full length Rgs12 (Accession: NM_173402.2)

22 cDNA was cloned into the p3XFLAG-myc-CMV-26 expression vector (Sigma-Aldrich, St. Louis,

$23 \mathrm{MO}, \mathrm{USA})$. Briefly, Hindll sites were incorporated into both termini of the Rgs12 cDNA using

24 restriction-site-generating PCR, and the restriction sites were used to insert the Rgs12

25 sequence into the expression vector containing an N-terminus FLAG tag sequence (Flag-

26 Rgs12). The primer walking method was used to validate the correct directionality of the insert. 
1 Additionally, a vector expressing C-terminus His-tagged Rgs12 (Rgs12-His) was generated by

2 subcloning the Rgs12 cDNA into the pcDNA3.1(+)-c-His vector (Genscript, Piscataway, NJ,

3 USA). Hindll and EcoRV sites were introduced by PCR and the restriction sites were used to

4 insert Rgs12 into the His vector. All vector constructs were confirmed by DNA sequencing

5 (Eurofins Genomics, Louisville, KY, USA).

Stable Transfection. RAW264.7 cells were seeded at $2 \times 10^{6}$ cells per 6 -well and transfected using FuGENE HD reagent (Promega, Madison, WI, USA) according to manufacturer's

9 instruments at a 1:3 DNA to transfection reagent ratio. After 48 hours post-transfection, cells

10 were treated with $0.4 \mathrm{mg} / \mathrm{mL}$ geneticin (G418, Thermo Fisher Scientific, Waltham, MA, USA) for

112 weeks until antibiotic-resistant colonies are formed. Stably transfected cells were thereafter

12 maintained in media containing $0.4 \mathrm{mg} / \mathrm{mL} \mathrm{G} 418$.

In Vitro Osteoclastogenesis and TRAP staining. The vector encoding the recombinant mRANKL-His (K158-D316) construct and a modified E. Coli strain Origami B(DE3) cells (EMD Millipore, Billercica, MA, USA) co-expressing chaperone proteins that was used to express the

17 recombinant RANKL were gifts from Dr. Ding Xu (University at Buffalo, School of Dental Medicine, Buffalo, NY). The protocol for expressing and purifying mRANKL-His was described previously (40). Endotoxins were removed using the Pierce High Capacity Endotoxin Removal

20 Resin (Thermo Fisher Scientific, Waltham, MA, USA). The M-CSF-producing cell line CMG14-

2112 was a gift from Dr. Sunao Takeshita (National Center for Geriatrics and Gerontology, Obu,

22 Japan). M-CSF production and bioassay were performed as previously described (41).

24 BMMs were obtained from the tibia and femur of 8-weeks-old C57BL/6J mice as described 25 previously (13). BMMs were seeded at $2 \times 10^{6}$ cells per 24 -well and stimulated with $100 \mathrm{ng} / \mathrm{mL}$

26 RANKL and $20 \mathrm{ng} / \mathrm{mL}$ M-CSF for 5 days to generate mature OCs. RAW264.7 cells were seeded 
1 at $1.35 \times 10^{4}$ cells per 24-well and stimulated with $100 \mathrm{ng} / \mathrm{mL}$ RANKL for 5 days. Prior to fixing

2 and staining, RAW264.7-derived OCs were rinsed thoroughly with PBS to remove mononuclear

3 cells that tend to obscure OCs during imaging. TRAP staining was performed using the acid

4 phosphatase, leukocyte (TRAP) kit (Sigma-Aldrich, St. Louis, MO, USA). Cells were imaged

5 using the Cytation 5 Cell Imaging Multi-Mode Reader (BioTek, Winooski, VT, USA) using the

6 montage function. Osteoclasts were quantified by counting the number of $\mathrm{TRAP}^{+}$,

7 multinucleated cells (MNCs, $\geq 3$ nuclei/cell) per well. Average osteoclast area was determined by

8 measuring total $\mathrm{TRAP}^{+}$area using the ImageJ software (US National Institute of Health,

9 Bethesda, MA, USA) and dividing the value by total osteoclast number.

Reverse Transcription and Quantitative PCR. Total RNA was isolated from cultured BMMs and OCs using Trizol reagent (Invitrogen, Carlsbad, CA, USA) following manufacturer's instructions. cDNA was reverse transcribed from $2 \mu \mathrm{g}$ total RNA using the RNA to cDNA EcoDry Premix kit (Clontech, Palo Alto, CA, USA). Primers were designed using Primer-BLAST (42)

17 and obtained from IDT (Integrated DNA Technologies, San Diego, CA, USA). Rgs12 (F: 5'AAGATCCATTCCCTAGTGACC-3', R: 5'-ACCTCCACTTTCCCACCCTG-3', 587 bp), Nrf2 (F: 5'-GCCCACATTCCCAAACAAGAT-3', R: 5'-CCAGAGAGCTATTGAGGGACTG-3', 172 bp), Keap1 (F: 5'-TGCCCCTGTGGTCAAAGTG-3', R: 5'-GGTTCGGTTACCGTCCTGC-3', 104 bp),

$21 \beta$-actin (F: 5'-CTAGGCACCAGGGTGTGAT-3', R: 5'-TGCCAGATCTTCTCCATG TC-3', 148

22 bp). qPCR was performed using the 2x SYBR Green qPCR Master Mix following manufacturer's

23 instructions (Bimake, Houston, TX, USA). All reactions were performed in triplicate and

24 normalized to the housekeeping gene $\beta$-actin. Data analysis was performed using the CTX

25 Maestro software (Bio-Rad, Hercules, CA, USA). 
1 Fluorescent actin cytoskeleton staining. Cells were starved in medium containing $1 \%$ fetal

2 bovine serum (FBS) and $5 \mathrm{ng} / \mathrm{mL}$ M-CSF for 16 hours then $0 \%$ FBS and $0 \mathrm{ng} / \mathrm{mL}$ M-CSF for 24

3 hours. Cytoskeletal reorganization was induced by introducing $50 \mathrm{ng} / \mathrm{mL} \mathrm{M}-\mathrm{CSF}$ in serum-free

4 medium for 5 minutes. Cells were fixed and the actin cytoskeleton was visualized by rhodamine

5 phalloidin staining.

Rac1-GTP pulldown assay. The Rac1-GTP pulldown assay was performed following manufacturer instructions in the Rac1 activation assay kit (Cytoskeleton, Denver, CO, USA).

ROS measurement. To measure ROS production, BMMs were seeded into black, glass-bottom

11 96-well plates and cultured with M-CSF for 48 hours until confluence. Cells were loaded with 20

$12 \mu \mathrm{M}$ 2'7'-dichlorofluorescein diacetate (DCFDA, Sigma) at $37^{\circ} \mathrm{C}$ for 30 minutes and washed

13 using PBS. The cells were swapped into complete phenol red-free MEM (Gibco) containing

14 RANKL/M-CSF. Fluorescence intensity was measured using the Cytation 5 plate reader

15 (BioTek) with excitation wavelength at $488 \mathrm{~nm}$ and emission wavelength at $535 \mathrm{~nm}$. Background

16 signals (cells not loaded with DCF-DA) were subtracted. Experiments were carried out in

17 quintuplicate wells.

Protein Extraction and Precipitation/ On-Pellet Digestion. Cells were harvested using ice-

20 cold lysis buffer (50 mM Tris-formic acid, $150 \mathrm{mM} \mathrm{NaCl}, 0.5 \%$ sodium deoxycholate, $1 \%$ SDS,

$212 \%$ NP-40, pH 8.0) with protease inhibitor (cOmplete, Mini, EDTA-free; Roche, Mannheim,

22 Germany). Samples were prepared for MS analysis using an established method $(17,43)$.

24 Liquid Chromatography-Tandem Mass Spectrometry Analysis. The "IonStar" LC-MS

25 experimental pipeline was developed and optimized in a previous study $(17,43)$. A 
1 stringent set of criteria including a low peptide and protein false discovery rate (FDR) of

$2<1 \%$ and $\geq 2$ peptides per protein was used for protein identification. An ion current-

3 based quantification method (IonStar processing pipeline) was described previously (17,

4 43).

5

6 Bioinformatics Analysis. Ingenuity Pathway Analysis (Qiagen, Redwood City, CA, USA) was

7 used to perform gene ontology enrichment analysis. Hierarchical clustering analysis and heat

8 map visualizations were performed using the agnes function in R Package cluster and ggplot2

9 with the viridis color palette, respectively.

11 Immunofluorescence. For the Nrf2 nuclear translocation experiment, BMMs were cultured on

12 coverslips and treated with RANKL and M-CSF for $72 \mathrm{~h}, 5 \mathrm{mM}$ NAC for $16 \mathrm{~h}$, or $50 \mu \mathrm{M}$ tBHP for

$1316 \mathrm{~h}$. Coverslips were fixed with 4\% paraformaldehyde solution in PBS for 10 minutes at room

14 temperature and permeabilized using $0.1 \%$ Triton $\mathrm{X}-100$ for 5 minutes at room temperature.

15 Coverslips were blocked using Image-iT FX signal enhancer (Thermo Fisher Scientific) for 1

16 hour at room temperature, stained with the primary antibody in $1 \% \mathrm{BSA} / \mathrm{TBST}$ overnight at $4{ }^{\circ} \mathrm{C}$,

17 and stained with the secondary antibody for 1 hour at room temperature. 4,6-diamidino-2-

18 phenylindole (DAPI) (Sigma) was used as a counterstain for nuclei. The coverslips were

19 mounted using ProLong Gold antifade mountant (Thermo) and images were obtained using a

20 fluorescence microscope (Leica, Wetzlar, Germany).

22 Western blotting. For experiments studying the Keap1-Nrf2 pathway, cells were cultured in 6-

23 well plates and pre-treated with the indicated concentrations of tBHQ or $25 \mu \mathrm{M} M G-132$ for $4 \mathrm{~h}$.

24 For MAPK and NFKB activation experiments, stable-transfected RAW264.7 cells were cultured

25 in 6-well plates and starved in serum-free medium containing $5 \mathrm{mM} \mathrm{NAC}$ for $16 \mathrm{~h}$. Cells were 
1 subsequently induced with RANKL (200 ng/mL) and M-CSF (100 ng/mL) for the indicated times.

2 Western blotting was performed as described previously (44). The primary antibodies used in

3 this study were as follows: Nrf2 (H-300) and Nrf2 (C-20) (1:100, Santa Cruz Biotechnology,

4 Dallas, TX, USA), Keap1 (E-20) (1:100, SCBT), phospho-p38 (Thr180/Tyr182) (1:1000, Cell

5 Signaling Technology), p38 (1:1000, CST), phospho-ERK1 (Thr202/Tyr204) + ERK2

6 (Thr186/Tyr187) (1:100, Abcam), ERK1/2 (1:1000, CST), phospho-NFkB p65 (Ser536) (1:1000,

7 CST), NFKB p65 (1:1000, CST), and $\beta$-actin (1:4000, SCBT). Densitomety analysis was

8 performed using ImageJ(45) and normalized to the $\beta$-actin signal. Relative phosphorylation of

9 was presented as the ratio between the phosphorylated normalized to the non-

10 phosphorylated/total protein. NAC, tBHQ, and tBHP were obtained from Sigma-Aldrich (St.

11 Louis, MO, USA), and MG-132 was obtained from Selleck Chemicals (Houston, TX, USA).

\section{ACKNOWLEDGMENTS}

14 This work was supported by the National Institute of Arthritis and Musculoskeletal and Skin

15 Diseases (NIAMS, AR061052), the National Institute of Aging (NIA, AG048388) awarded to Dr.

16 Shuying Yang. We would like to thank Dr. Ding Xu and Dr. Sunao Takeshita for their generous

17 gifts of the mRANKL-His (K158-D316) vector and CMG14-12 cell line, respectively.

\section{REFERENCES}

20 1. Amin S, Achenbach SJ, Atkinson EJ, Khosla S, Melton LJ, 3rd. Trends in fracture incidence: a population-based study over 20 years. J Bone Miner Res. 2014;29(3):581-

23 2. Neubig RR, Siderovski DP. Regulators of G-protein signalling as new central nervous system drug targets. Nat Rev Drug Discov. 2002;1(3):187-97.

3. Keinan D, Yang S, Cohen RE, Yuan X, Liu T, Li YP. Role of regulator of G protein signaling proteins in bone. Front Biosci (Landmark Ed). 2014;19:634-48. 
4. Snow BE, Brothers GM, Siderovski DP. Molecular cloning of regulators of G-protein signaling family members and characterization of binding specificity of RGS12 PDZ domain. Methods Enzymol. 2002;344:740-61.

5. Sambi BS, Hains MD, Waters CM, Connell MC, Willard FS, Kimple AJ, et al. The effect of RGS12 on PDGFbeta receptor signalling to p42/p44 mitogen activated protein kinase in mammalian cells. Cell Signal. 2006;18(7):971-81.

6. Snow BE, Hall RA, Krumins AM, Brothers GM, Bouchard D, Brothers CA, et al. GTPase activating specificity of RGS12 and binding specificity of an alternatively spliced PDZ (PSD-95/Dlg/ZO-1) domain. J Biol Chem. 1998;273(28):17749-55.

7. Willard MD, Willard FS, Li X, Cappell SD, Snider WD, Siderovski DP. Selective role for RGS12 as a Ras/Raf/MEK scaffold in nerve growth factor-mediated differentiation.

EMBO J. 2007;26(8):2029-40.

13 8. Schiff ML, Siderovski DP, Jordan JD, Brothers G, Snow B, De Vries L, et al. Tyrosine-

9. Callaway DA, Jiang JX. Reactive oxygen species and oxidative stress in osteoclastogenesis, skeletal aging and bone diseases. J Bone Miner Metab. 2015;33(4):359-70. kinase-dependent recruitment of RGS12 to the N-type calcium channel. Nature.

10. Lee NK, Choi YG, Baik JY, Han SY, Jeong DW, Bae YS, et al. A crucial role for reactive

12. Bartell SM, Kim HN, Ambrogini E, Han L, lyer S, Serra Ucer S, et al. FoxO proteins restrain osteoclastogenesis and bone resorption by attenuating $\mathrm{H} 2 \mathrm{O} 2$ accumulation. Nat Commun. 2014;5:3773.

14. Abram $\mathrm{CL}$, Roberge $\mathrm{GL}$, $\mathrm{Hu}$ Y, Lowell CA. Comparative analysis of the efficiency and specificity of myeloid-Cre deleting strains using ROSA-EYFP reporter mice. J Immunol

32 15. Clausen BE, Burkhardt C, Reith W, Renkawitz R, Forster I. Conditional gene targeting in macrophages and granulocytes using LysMcre mice. Transgenic Res. 1999;8(4):26577. 
16. Lees RL, Sabharwal VK, Heersche JN. Resorptive state and cell size influence intracellular $\mathrm{pH}$ regulation in rabbit osteoclasts cultured on collagen-hydroxyapatite films. Bone. 2001;28(2):187-94.

17. Shen X, Shen S, Li J, Hu Q, Nie L, Tu C, et al. IonStar enables high-precision, lowmissing-data proteomics quantification in large biological cohorts. Proc Natl Acad Sci U S A. 2018;115(21):E4767-E76.

18. Venugopal R, Jaiswal AK. Nrf1 and Nrf2 positively and C-Fos and Fra1 negatively

19. Itoh K, Chiba T, Takahashi S, Ishii T, Igarashi K, Katoh Y, et al. An Nrf2/small Maf regulate the human antioxidant response element-mediated expression of $\mathrm{NAD}(\mathrm{P}) \mathrm{H}$ :quinone oxidoreductase1 gene. Proc Natl Acad Sci U S A.

21. Kanzaki H, Shinohara F, Kajiya M, Kodama T. The Keap1/Nrf2 protein axis plays a role

22. Stewart D, Killeen E, Naquin R, Alam J. Degradation of the transcription factor Nrf2, via the ubiquitin-proteasome pathway and stabilization by cadmium. Faseb J. 2004;18(8):C71-C.

24. Itoh K, Wakabayashi N, Katoh Y, Ishii T, O'Connor T, Yamamoto M. Keap1 regulates

26. Abiko Y, Miura T, Phuc BH, Shinkai Y, Kumagai Y. Participation of covalent

23. Zhang DD, Hannink M. Distinct cysteine residues in Keap1 are required for Keap1dependent ubiquitination of Nrf2 and for stabilization of Nrf2 by chemopreventive agents and oxidative stress. Mol Cell Biol. 2003;23(22):8137-51. both cytoplasmic-nuclear shuttling and degradation of Nrf2 in response to electrophiles. Genes Cells. 2003;8(4):379-91.

25. Kensler TW, Wakabayashi N, Biswal S. Cell survival responses to environmental stresses via the Keap1-Nrf2-ARE pathway. Annu Rev Pharmacol Toxicol. 2007;47:89116. modification of Keap1 in the activation of Nrf2 by tert-butylbenzoquinone, an electrophilic metabolite of butylated hydroxyanisole. Toxicol Appl Pharmacol. 2011;255(1):32-9. 
1 27. Hyeon $S$, Lee $H$, Yang $Y$, Jeong W. Nrf2 deficiency induces oxidative stress and promotes RANKL-induced osteoclast differentiation. Free Radic Biol Med. 2013;65:789-99.

4 28. $\mathrm{Ha} H$, Kwak HB, Lee SW, Jin HM, Kim HM, Kim HH, et al. Reactive oxygen species mediate RANK signaling in osteoclasts. Exp Cell Res. 2004;301(2):119-27.

29. Kansanen E, Kuosmanen SM, Leinonen H, Levonen AL. The Keap1-Nrf2 pathway: Mechanisms of activation and dysregulation in cancer. Redox Biol. 2013;1:45-9.

30. Segeletz S, Hoflack B. Proteomic approaches to study osteoclast biology. Proteomics. 2016;16(19):2545-56.

31. Rotival M, Ko JH, Srivastava PK, Kerloc'h A, Montoya A, Mauro C, et al. Integrating phosphoproteome and transcriptome reveals new determinants of macrophage multinucleation. Mol Cell Proteomics. 2015;14(3):484-98.

34. Nguyen T, Nioi P, Pickett CB. The Nrf2-antioxidant response element signaling

35. Nguyen T, Sherratt PJ, Nioi P, Yang CS, Pickett CB. Nrf2 controls constitutive and

38. Yang S, Li YP, Liu T, He X, Yuan X, Li C, et al. Mx1-cre mediated Rgs12 conditional 
39. Yuan X, Cao J, Liu T, Li YP, Scannapieco F, He X, et al. Regulators of G protein signaling 12 promotes osteoclastogenesis in bone remodeling and pathological bone loss. Cell Death Differ. 2015;22(12):2046-57.

4 40. Li M, Yang S, Xu D. Heparan Sulfate Regulates the Structure and Function of

41. Takeshita S, Kaji K, Kudo A. Identification and characterization of the new osteoclast progenitor with macrophage phenotypes being able to differentiate into mature osteoclasts. J Bone Miner Res. 2000;15(8):1477-88.

9 42. Ye J, Coulouris G, Zaretskaya I, Cutcutache I, Rozen S, Madden TL. Primer-BLAST: a tool to design target-specific primers for polymerase chain reaction. BMC

12 43. Shen X, Shen S, Li J, Hu Q, Nie L, Tu C, et al. An IonStar Experimental Strategy for MS1 Ion Current-Based Quantification Using Ultrahigh-Field Orbitrap: Reproducible, 2017;16(7):2445-56.

16 44. Yuan X, Cao J, He X, Serra R, Qu J, Cao X, et al. Ciliary IFT80 balances canonical versus non-canonical hedgehog signalling for osteoblast differentiation. Nat Commun. 2016;7:11024.

45. Schindelin J, Arganda-Carreras I, Frise E, Kaynig V, Longair M, Pietzsch T, et al. Fiji: an open-source platform for biological-image analysis. Nat Methods. 2012;9(7):676-82. 

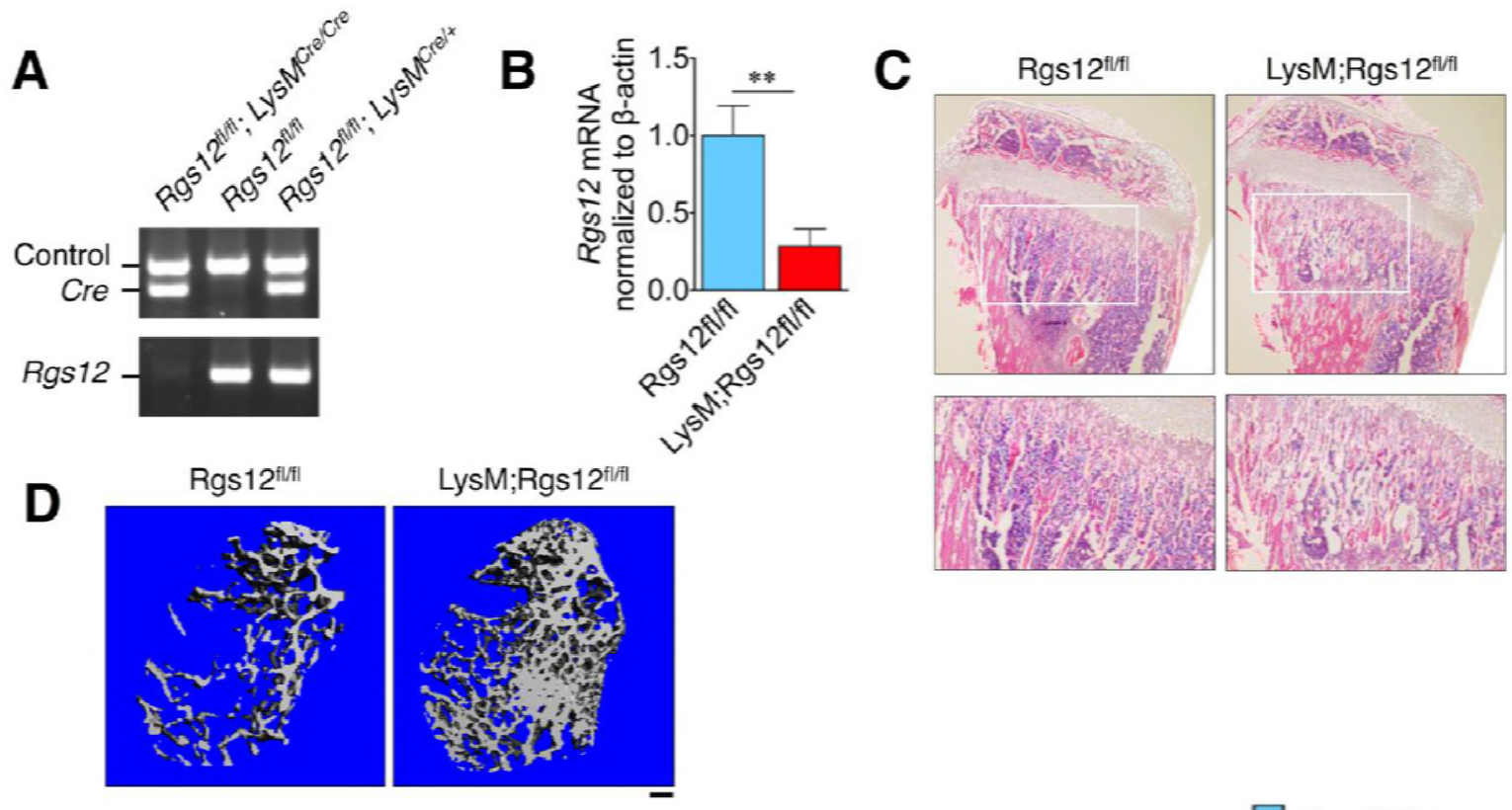

E

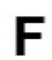

G
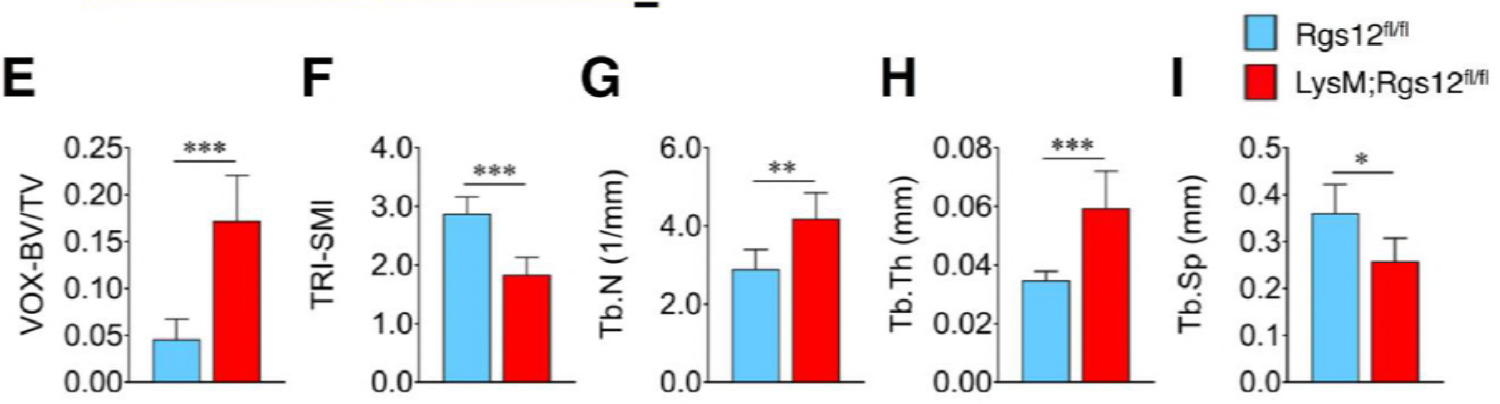

Figure 1. Rgs12-deficient mice exhibit increased trabecular bone mass. (A) PCR of splenic genomic DNA amplified the deletion allele of Rgs12 in LysM;Rgs12 $1 /$ /fli mice. (B) qPCR analysis of Rgs12 mRNA levels normalized to $\beta$-actin in BMMs obtained from Rgs $12^{\mathrm{fl} / \mathrm{fl}}$ and LysM;Rgs12 $1 /$ flf mice. Results are means $\pm \mathrm{SD}\left(n=3,{ }^{* *} p<0.01\right) .(\mathrm{C})$ Hematoxylin and eosin staining of the proximal tibias and (D) Representative 3D micro-computed tomographay (microCT) images of the trabecular bone of femurs obtained from 8-weeks-old Rgs $12^{f / / f l}$ and LysM;Rgs12 $12^{f / f l}$ mice. Scale bar on $(D)=200 \mu \mathrm{m}$. (E-I). Quantitative micro-CT measurements of

9 femur bone morphology and microarchitecture. Results are means $\pm \mathrm{SD}\left(n=4 ;{ }^{*} p<0.05\right.$, $\left.{ }^{* *} p<0.01,{ }^{* * *} p<0.001\right)$. VOX-BV/TV, bone volume to tissue volume (voxel count); TRI-SMI,

11 structure model index; Tb.Th, trabecular thickness; Tb.N, trabecular number; Tb.Sp, trabecular 12 separation. 


\section{Figure 1-source data 1}

3 This Excel sheet contains the micro-CT numerical data and summary statistics represented in

4 Fig. 1E-I.

A

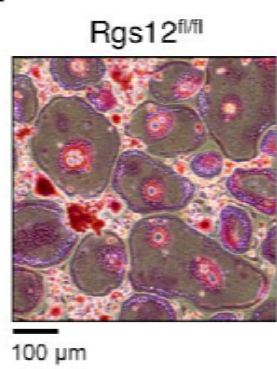

D

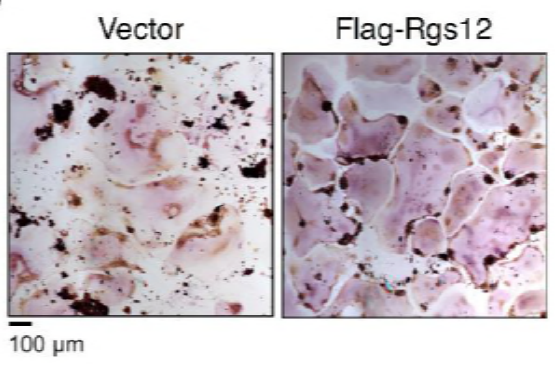

LysM;Rgs $12^{\text {flifl }}$

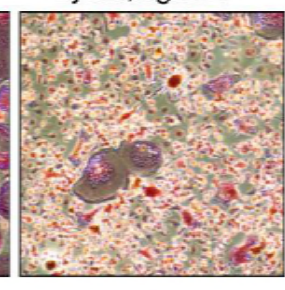

E

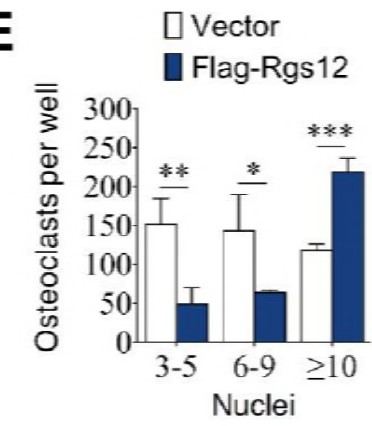

C

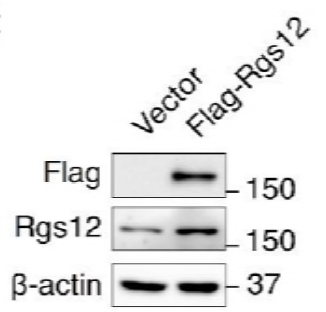

$\mathbf{F}$

$\square$ vector

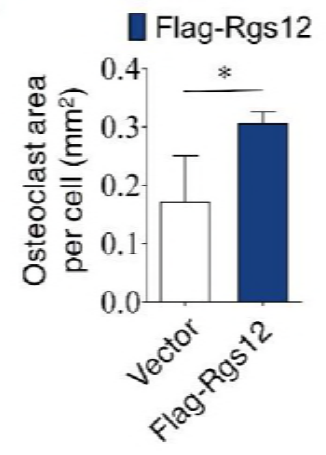

Figure 2. Rgs12 is essential for osteoclast differentiation. (A) TRAP-stained osteoclasts differentiated from BMMs isolated from Rgs $12^{t / f|l|}$ and LysM;Rgs12 $2^{t / f l t}$ mice. (B) Number of TRAP-

9 positive and multinucleated ( $\geq 3$ nuclei) osteoclasts from Rgs $12^{f / f / l}$ and LysM;Rgs $12^{\mathrm{ft/fl}}$ BMMs $\left(n=4,{ }^{\star} P<0.05,{ }^{* \star} P<0.01,{ }^{* \star *} P<0.001\right)$.

(C) Immunoblot to verify Rgs12 overexpression in

11 RAW264.7 cells transfected with a vector carrying a recombinant N-terminus FLAG-tagged

12 Rgs12 gene (Flag-Rgs12). RAW264.7 cells transfected with the empty vector was used as a

13 negative control. (D) TRAP-stained osteoclasts derived from RAW264.7 cells transfected with

14 an empty vector or Flag-Rgs12. (E) Number of TRAP-positive and multinucleated ( $\geq 3$ nuclei) 
1 osteoclasts from vector- and Flag-Rgs12-transfected RAW264.7 cells $\left(n=3,{ }^{*} P<0.05,{ }^{* \star} P<0.01\right.$,

$\left.2^{* \star *} P<0.001\right)$. (F) OC size was estimated by quantifying the surface area of osteoclasts

3 containing $10+$ nuclei, which was normalized to the number of osteoclasts with $10+$ nuclei $(n=3$,

$\left.4 \quad{ }^{*} P<0.05\right)$. TRAP, tartrate-resistant acid phosphatase.

5

A

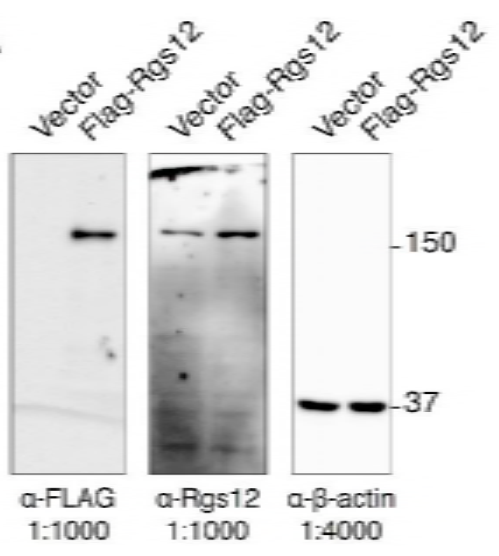

Figure 2-figure supplement 1. Complete western blots used for Figure 2C. 
A

\begin{tabular}{|lc|}
\hline Total proteins ID'd & 3,714 \\
Upregulated & 83 \\
Downregulated & 61 \\
\hline
\end{tabular}

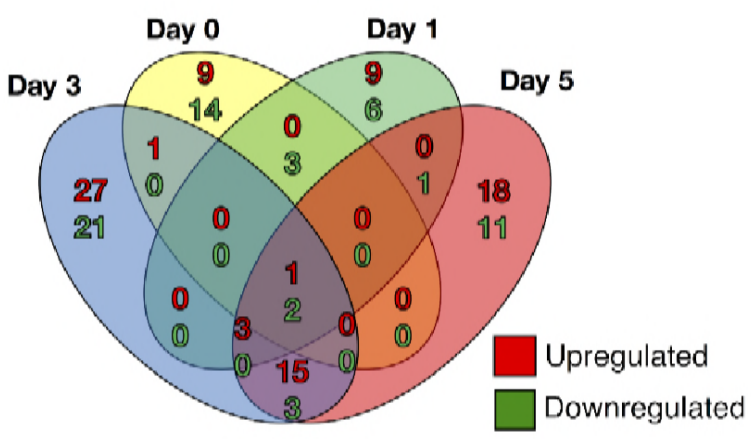

B
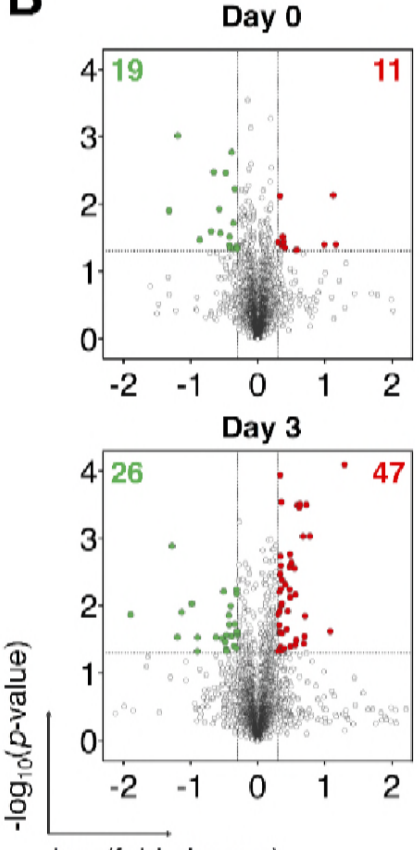

$\log _{2}$ (fold-change)

D

\begin{tabular}{lcccc} 
& \multicolumn{4}{c}{$\begin{array}{c}\text { Osteoclast Marker Proteins } \\
\log _{2} \text { (fold-change) }\end{array}$} \\
Protein & 0d & 1d & 3d & 5d \\
\cline { 2 - 5 } Mmp9 & 0.1 & 0.3 & -0.3 & -0.3 \\
Trap & -0.1 & 0.1 & -0.2 & -0.2 \\
Nfatc1 & 0.1 & 0.1 & -0.2 & -0.2 \\
Atp6v0d2 & 0.0 & 0.0 & -0.4 & -0.3 \\
Itgb3 & 0.3 & 0.0 & -0.3 & 0.0 \\
Cah & 0.3 & 0.5 & -0.2 & -0.3 \\
Oscar & 0.2 & 0.7 & -0.3 & -0.1
\end{tabular}

Shaded values are statistically significant.

E

\section{C \\ Gene Ontology (GO) Enrichment Analysis}

Days

Canonical Pathways $013 \quad 3$

*Production of Reactive Oxygen Species

NFAT Signaling

PI3K Signaling

tRANK Signaling in Osteoclasts

Role of Macrophages in RA

tRole of Osteoclasts in RA *Superoxide Radicals Degradation

Pentose Phosphate Pathway FcRy Receptor-mediated Phagocytosis

NF-kB Activation

Paxillin Signaling

IL-8 Signaling

Leukocyte Extravasation Signaling

"Thioredoxin Pathway

*NRF2-mediated Stress Response

Oxidative Phosphorylation

Sirtuin Signaling Pathway

Mitochondrial Dysfunction

Heme Biosynthesis

*Glutathione Redox Reactions I

"Glutathione Redox Reactions II

Rac Signaling Integrin Signaling

Autophagy

Phagosome Maturation Iron Homeostasis Histidine Degradation VI Urea Cycle

Citrulline Metabolism Histidine Degradation III

*ROS Homeostasis tosteoclast Differentiation

\section{NRF2-regulated Antioxidant Proteins} $\log _{2}$ (fold-change)

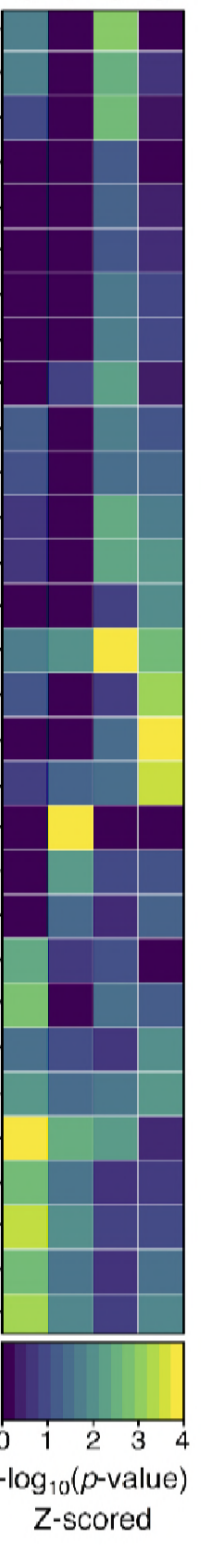

Protein

Prdx1

\begin{tabular}{cccc} 
0d & 1d & 3d & 5d \\
\hline-0.1 & 0.1 & 0.7 & 0.6
\end{tabular}

Prdx4

$\begin{array}{llll}0.0 & 0.0 & 0.7 & 0.6\end{array}$

Cata

$\begin{array}{llll}-0.1 & 0.0 & 0.4 & 0.2\end{array}$

Trxi1

$\begin{array}{llll}0.1 & 0.1 & 0.3 & 0.2\end{array}$

Trxr2

$\begin{array}{llll}0.1 & 0.1 & -0.1 & 0.3\end{array}$

Gshr

$\begin{array}{llll}0.3 & 0.4 & 0.8 & 0.7\end{array}$

Nqo1

$\begin{array}{llll}-0.1 & 0.5 & 0.3 & 0.0\end{array}$

Shaded values are statistically significant. 
2 Figure 3. Proteomics analysis identified an increased expression of Nrf2-dependent antioxidant

3 proteins in Rgs12-deficient osteoclast precursors. (A) Venn diagram summarizing the

4 distribution of proteins that were significantly altered in LysM;Rgs $12^{\mathrm{fl} / \mathrm{fl}} \mathrm{BMMs}$ as compared to

5 Rgs12 ${ }^{f / / f l}$ BMMs at $0,1,3$, and 5 days of OC differentiation. (B) Volcano plots depicting protein

6 expression changes in LysM;Rgs12 $12^{f / f l}$ BMMs as compared to Rgs12 $2^{f / f l}$ BMMs. Optimized cutoff

7 thresholds for significantly altered proteins was set at $1.3 \log _{2}$-tranformed ratios and $p$-value $<$

8 0.05. Data are means \pm SD. Student's $t$ test was performed to compare Rgs $12^{\mathrm{fl} / \mathrm{fl}}$ and

9 LysM;Rgs12 $12^{f / f l}$ BMMs at each time point $(n=3)$. (C) Gene ontology (GO) enrichment analysis to

10 identify canonical pathways corresponding to the significantly altered proteins. For visualization

11 purposes, the color intensity in the heat map diagram indicates the significance of GO term

12 enrichment, presented as -log10(p-value). Hierarchical clustering analysis was used to group

13 GO terms based on the $p$-value of enrichment. (D, E) The expression of OC marker proteins

14 and Nrf2-regulated antioxidant proteins in LysM;Rgs12 $2^{\mathrm{fl} / f 1}$ versus Rgs12 $2^{\mathrm{fl} / \mathrm{fl}}$ BMMs. Mmp9,

15 metalloproteinase-9; Trap, tartrate-resistant acid phosphatase; Nfatc1, nuclear factor of

16 activated T cells, cytoplasmic 1; Atp6v0d2, ATPase $\mathrm{H}^{+}$transporting V0 subunit D2; Itgb3,

17 integrin $\beta 3$; Prdx, peroxiredoxin; Cata, catalase; Trxr, thioredoxin; Gshr, glutathione reductase;

$18 \mathrm{Nqo1}, \mathrm{NAD}(\mathrm{P}) \mathrm{H}$ dehydrogenase quinone 1.

20 Figure 3-source data 1. Table of proteomics data presented in Fig. 3A,B,D,E. Quantitative

21 proteomics analysis of 3,714 proteins in Rgs12 knockout versus wild-type OCs at different

22 timepoints of differentiation. Statistical comparisons between groups were evaluated by

23 Student's t-test $(P<0.05, n=3)$. 
A

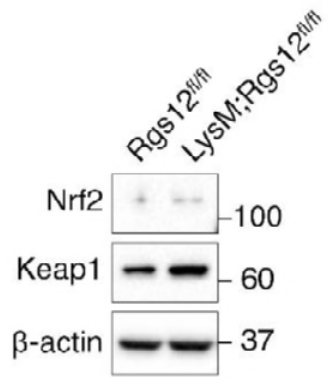

B

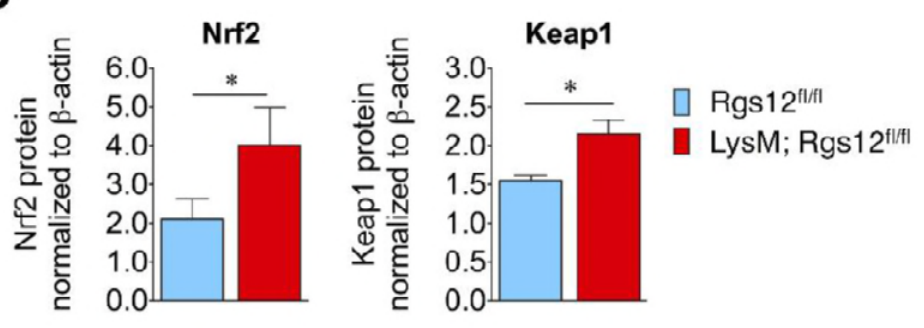

C

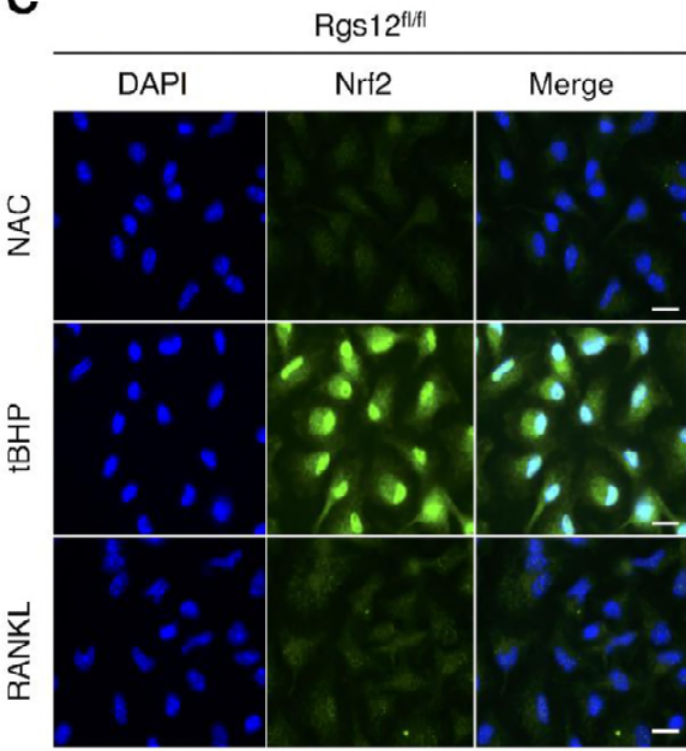

D

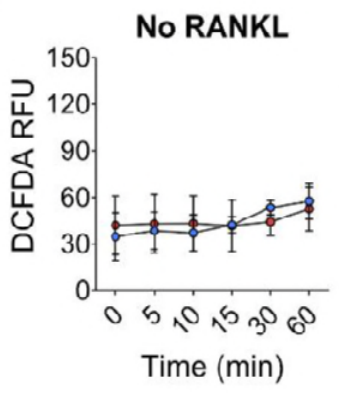

RANKL

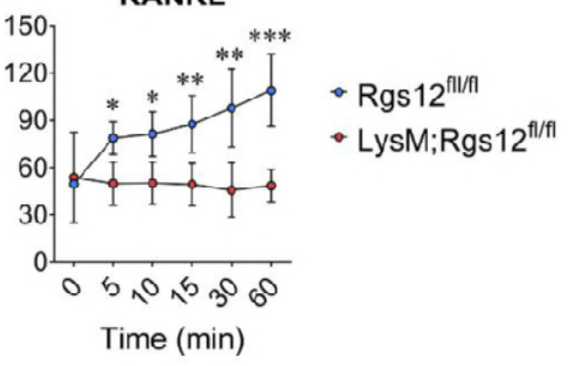

2 Figure 4. Increased Nrf2 activation and expression of antioxidant proteins in Rgs12-deficient

3 osteoclast precursors. (A-F) Nrf2 immunofluorescence staining in Rgs1 $2^{t / / f l}$ and LysM;Rgs1 $2^{\mathrm{t} / / 1}$

4 BMMs differentiated with M-CSF and RANKL for $72 \mathrm{~h}$. (C, D). As a negative control for Nrf2

5 nuclear translocation, cells were treated with the antioxidant compound NAC ( $5 \mathrm{mM}, 16 \mathrm{~h})$ to

6 suppress cellular ROS. (E, F) Conversely, as a positive control for Nrf2 nuclear translocation,

7 cells were treated with the peroxidase tBHP $(50 \mu \mathrm{M}, 16 \mathrm{~h})$ to induce oxidative stress. $(G, H)$ 
1 Immunoblot of Nrf2 and Keap1 protein levels in Rgs12 $2^{f / f l}$ and LysM;Rgs12 $2^{f / f l}$ BMMs treated with

2 RANKL for $72 \mathrm{~h}$. Densitometry analysis was performed on bands and normalized to $\beta$-actin

$3\left(n=3,{ }^{*} p<0.05\right)$. (I) Induction of ROS levels in Rgs12f//fl and LysM;Rgs12f//fl BMMs differentiated

4 for $72 \mathrm{~h}$, kept in serum-free medium for $6 \mathrm{~h}$, and stimulated with RANKL for the indicated times.

5 ROS levels were measured using the DCFDA fluorescence method. Data are means \pm SD $(n=5$,

$\left.6{ }^{*} p<0.05,{ }^{* *} p<0.01,{ }^{* *} p<0.001\right)$. DAPI, 4,6-diamidino-2-phenylindole; NAC, N acetylcysteine;

7 tBHP, tert-butylhydroxyperoxide. ROS, reactive oxygen species. DCFDA, 2',7'-

8 dichlorofluorescin diacetate. RFU, relative fluorescence units. 


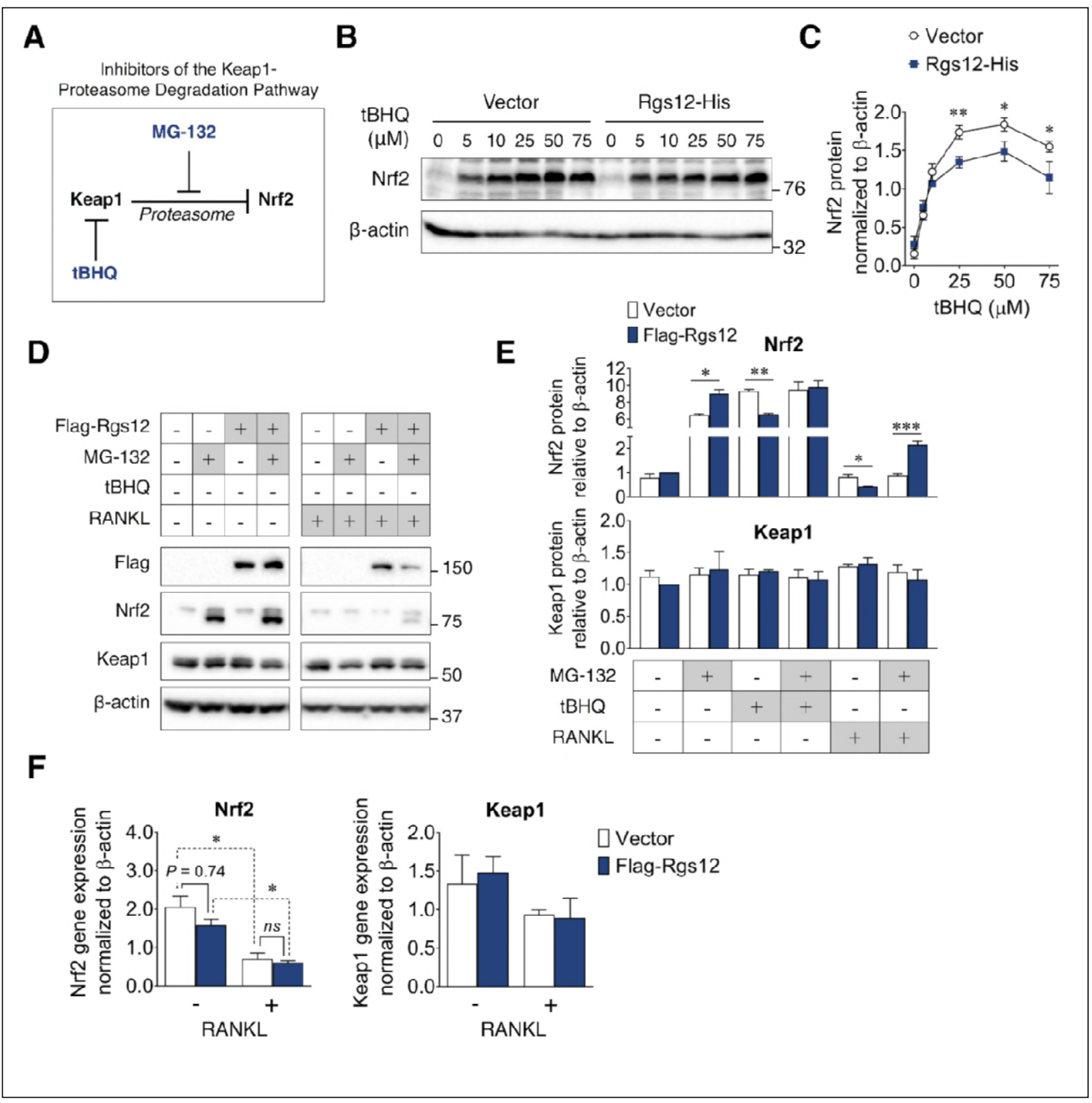

2 Figure 5. Suppression of Nrf2 protein levels by Rgs12 is dependent on the proteasome

3 degradation pathway. (A) Diagram summarizing the inhibitors of the Keap1-proteasome axis to

4 modulate Nrf2 protein levels. (B) RAW264.7 cells stably-transfected with Rgs12-His or empty

5 vector treated with increasing doses of tBHQ. (C) Nrf2 and Keap1 protein levels were quantified

6 by densitometry analysis and normalized to $\beta$-actin $\left(n=3,{ }^{*} p<0.05,{ }^{* *} p<0.01\right)$. (D) Western blot to

7 detect Nrf2 and Keap1 in RAW264.7 cells stably-transfected with empty vector or Flag-Rgs12. 
1 RAW264.7 cells were treated with a combination of RANKL (100 ng/mL, $72 \mathrm{~h}$ ) and the

2 proteasome inhibitor MG-132 (25 $\mu \mathrm{M}, 4 \mathrm{~h})$. (E) Nrf2 and Keap1 protein levels were quantified by

3 densitometry analysis and normalized to $\beta$-actin $\left(n=3,{ }^{*} p<0.05,{ }^{* *} p<0.01,{ }^{* \star *} p<0.001\right)$. (F) qPCR

4 analysis of Nrf2 and Keap1 transcript levels in RAW264.7 cells transfected with Rgs12-His or

5 empty vector. Data are means $\pm \mathrm{SD}$. Two-tailed $t$ test was performed $\left(n=3,{ }^{\star} P<0.05\right)$. tBHQ, tert-

6 butylhydroquinone. 
A

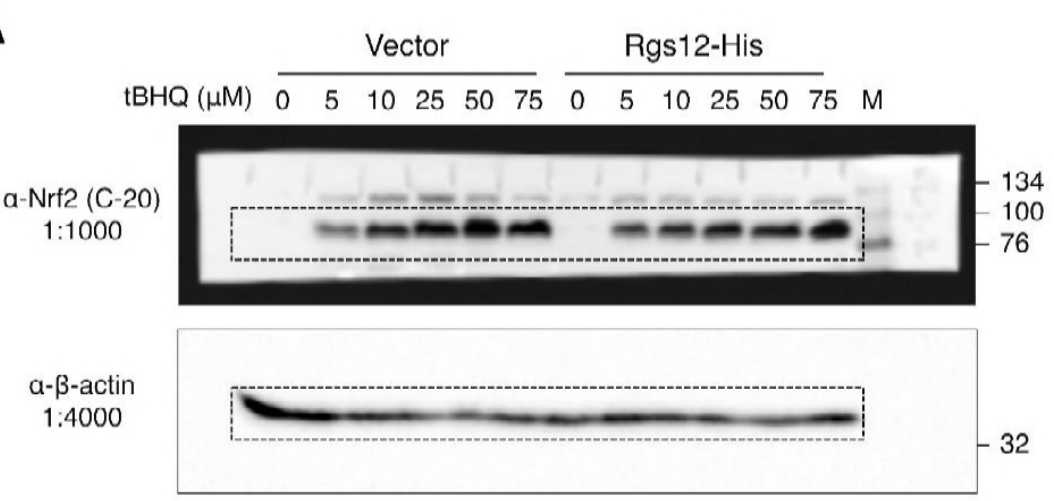

B
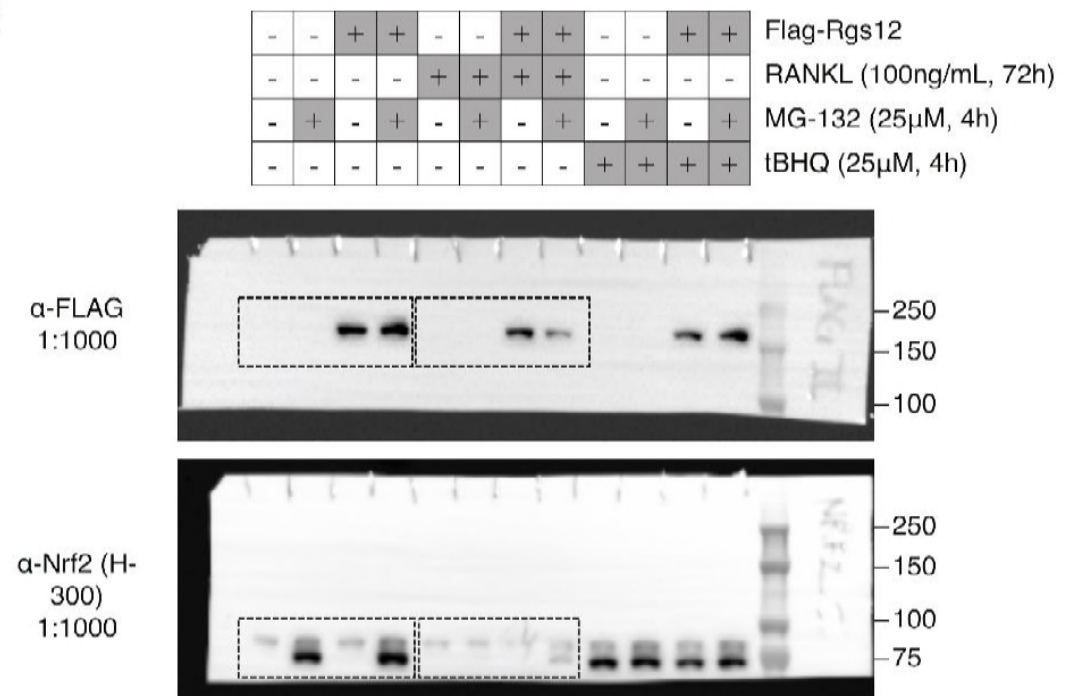

2 Figure 5-figure supplement 1. (A) Complete western blots used in Figure 5B. Sections

3 shown in Figure 5B are highlighted with dashed boxes. Transfected RAW264.7 cells were

4 induced with the indicated dosages of tBHQ for 4 h. (B) Complete western blots used in Figure

5 5D. Sections shown in Figure 5D are highlighted with dashed boxes. RAW264.7 cells stably- 
1 transfected with empty vector or Flag-Rgs12 were treated with the following: RANKL (100

$2 \mathrm{ng} / \mathrm{mL}, 72 \mathrm{~h}), \mathrm{MG}-132(25 \mu \mathrm{M}, 4 \mathrm{~h})$, and/or tBHQ (25 $\mu \mathrm{M}, 4 \mathrm{~h})$. tBHQ, tert-butylhydroquinone.
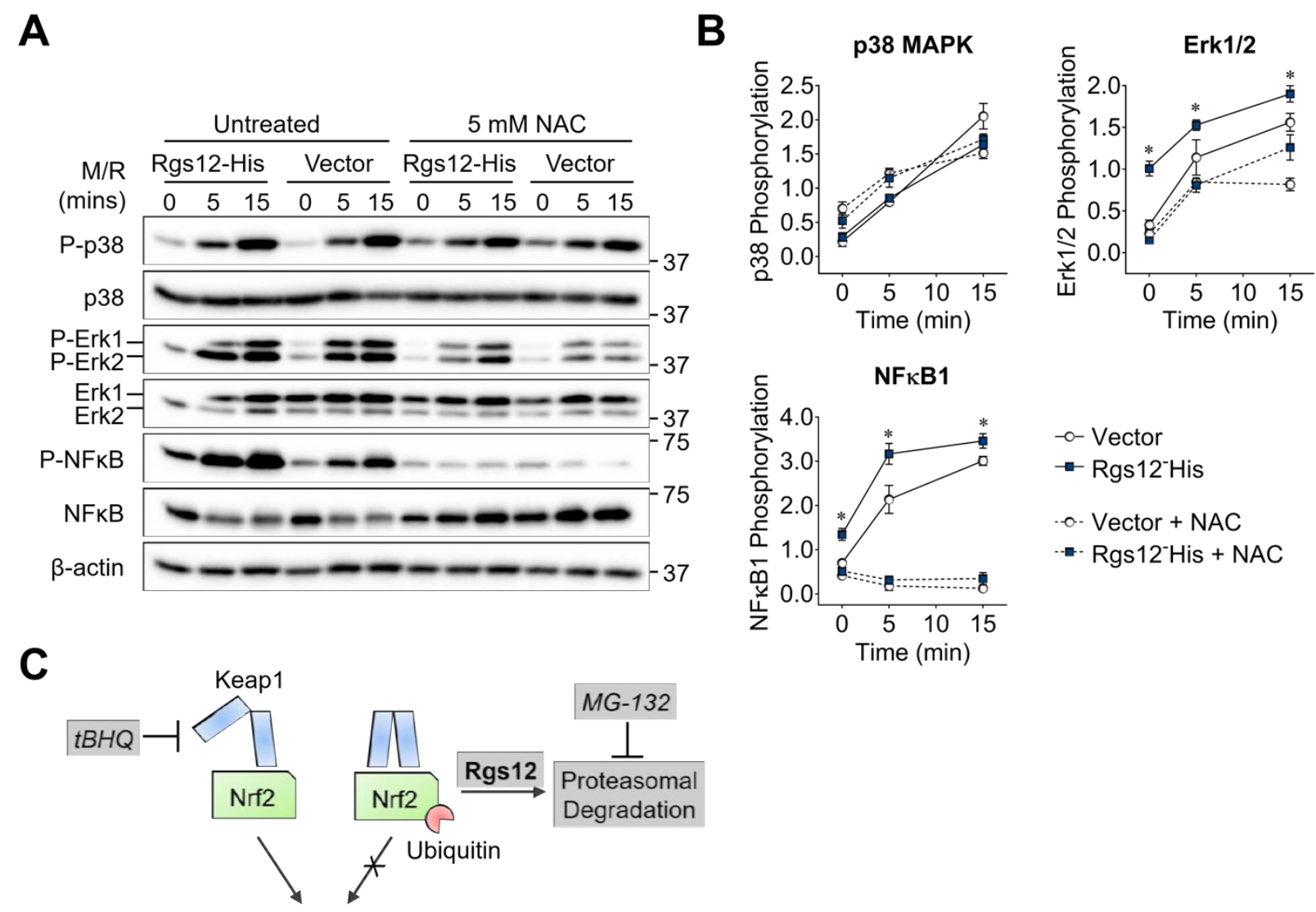

Phase II detoxification \& antioxidant enzymes

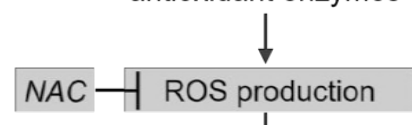

OC signaling \& differentiation

5 Figure 6. Rgs12-dependent activation of MAPK and NFkB was suppressed by antioxidants.

6 (A) Western blot detected phosphorylated or total p38, NFkB, and Erk1/2 in transfected

7 RAW264.7 cells induced with RANKL (200 ng/mL) and M-CSF (100 ng/mL) for the indicated

8 times. Cells were pretreated with NAC (5 mM, $4 \mathrm{~h})$ to suppress cellular reactive oxygen species.

9 (B) Band density was quantified by ImageJ and phosphorylated and unphosphorylation/total 
1 protein levels were normalized to $\beta$-actin. Relative phosphorylation are presented as the ratio

2 between the phosphorylated normalized to the nonphosphorylated/total protein. Two-tailed $t$

3 tests were used to compare vector and Rgs12-His groups ( $\left.n=3,{ }^{*} P<0.05\right)$. M/R, M-CSF and

4 RANKL. NAC, N-acetylcysteine. (C) Model of the role of Rgs12 in suppressing Nrf2 to promote

5 ROS and OC differentiation.

A

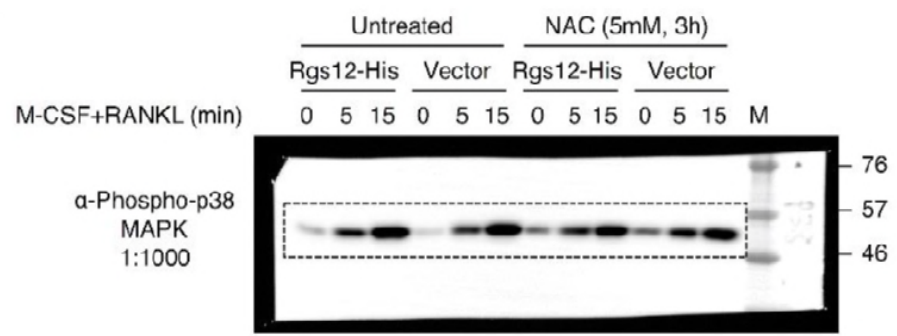

a-Phospho-p38 MAPK 1:1000

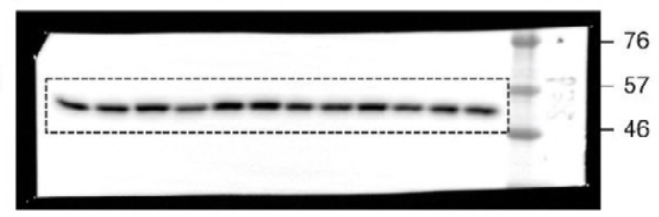

a-PhosphoNFKB1 (p65) 1:1000

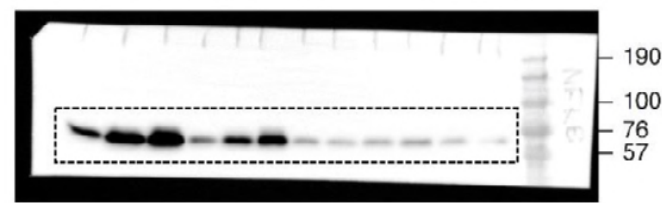

a-NFKB1 (p65)

$1: 1000$

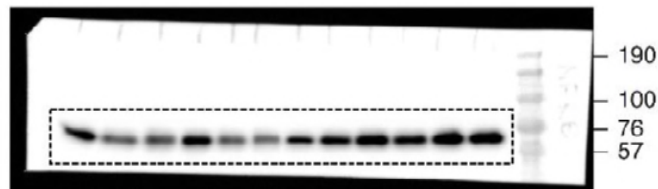

a-Phospho-

Erk1/2

1:1000
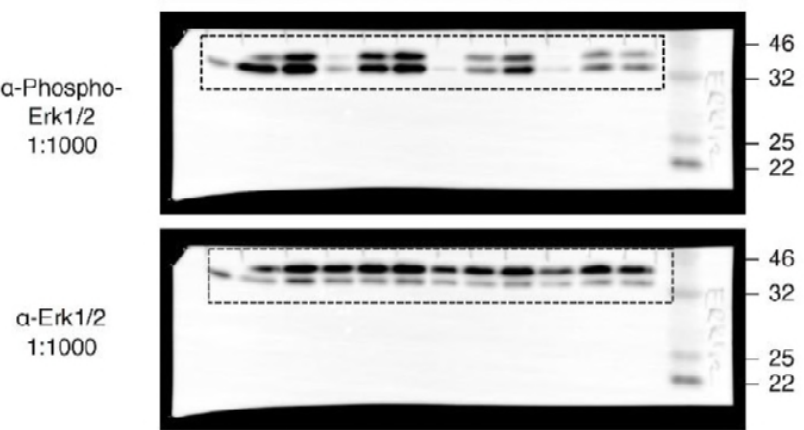

a- $\beta$-actin

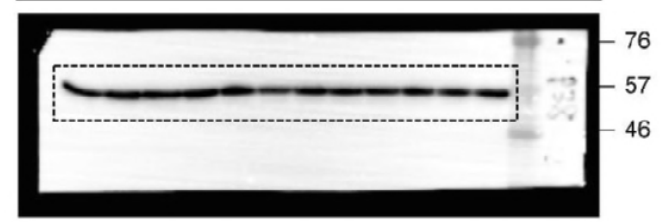


bioRxiv preprint doi: https://doi.org/10.1101/470542; this version posted November 14, 2018. The copyright holder for this preprint (which was not certified by peer review) is the author/funder, who has granted bioRxiv a license to display the preprint in perpetuity. It is made available under aCC-BY 4.0 International license.

1 Figure 6-figure supplement 1. (A) Complete western blots used in Figure 7A. Sections

2 shown in Figure 6C are highlighted with dashed boxes. 